\section{Differences in Palmoplantar Pustulosis and Psoriasis Vulgaris in Patients with Rheumatoid Arthritis or Ankylosing Spondylitis Treated with Biological Therapy}

\section{To the Editor:}

Biological treatment is an effective treatment for various chronic inflammatory diseases ${ }^{1,2}$. Nevertheless, reports of paradoxical immune-mediated inflammation induced by biologicals are increasing ${ }^{3}$. A paradoxical adverse event is defined as the development of inflammatory manifestations in patients after the initiation of biologicals, mostly tumor necrosis factor inhibitors (TNFi), normally used to treat such disorders ${ }^{3}$. Palmoplantar pustulosis (PPP) and psoriasis vulgaris (PV) are the most described paradoxical cutaneous adverse events ${ }^{4}$. Although studies show that PPP and PV are different entities, they are analyzed as 1 group of paradoxical cutaneous diseases ${ }^{5}$. Consequently, individual prevalence of paradoxical $\mathrm{PPP}$ and PV, and associated differences regarding the clinical consequence, are still unknown.

To study these differences, we retrospectively collected data from 2000 to 2015 of the Reade Rheumatology Registry, an observational cohort of patients with biological-treated ankylosing spondylitis (AS) and rheumatoid arthritis (RA). The 5 different TNFi, abatacept (ABA), tocilizumab (TCZ), and rituximab were included. Further, each patient received a multiple-choice questionnaire, from June 2016 until September 2016, regarding the occurrence of a skin disorder during their biological treatment. In patients with an unclear diagnosis, both patient and physician were contacted regarding the diagnosed cutaneous disorder. A skin event had to be confirmed by a physician. Only biologically naive patients were included, to study the clinical characteristics of PPP and PV. The study was approved by the ethics committee of the Slotervaart Hospital and Reade Amsterdam according to the declaration of Helsinki (ethics committee number: 6868) and all patients gave written informed consent.

A total of 1210 consecutive patients with RA (3792 person- yrs) and 289 consecutive patients with AS (1068 person-yrs) were included. Median time of followup in this cohort was 22 months [interquartile range (IQR) 6.0-56.0 mos] for patients with RA and 32 months (IQR 7.8-68.3 mos) for patients with AS. Development of PPP occurred in $8(0.66 \%)$ of the patients with RA and in $5(1.73 \%)$ of the patients with AS. PV developed in $12(0.99 \%)$ of the patients with RA and in $4(1.38 \%)$ of the patients with AS. New onset of PPP or PV occurred in, respectively, $72.7 \%$ and $77.8 \%$, during each patient's first biological treatment. New-onset PPP was found only in patients using TNFi. New-onset PV was found in patients using TNFi and 1 case of TCZ and 1 case of ABA.

As shown in Figure 1, median time to onset for PPP manifestations was 6 months (IQR 4.0-16.0) after start of biological treatment and for PV, 50 months (IQR 11.0-67.0; $\mathrm{p}=0.003$ ).

As for the clinical consequence, besides being treated with topical therapy and/or psoralen plus ultraviolet A therapy, $60 \%$ of the patients who developed PPP had to discontinue their biological treatment to be treated successfully. Of these patients, $80 \%$ developed an exacerbation of PPP on their second TNFi. In contrast to PV, topical therapy was sufficient and only $18 \%$ discontinued their biological treatment.

Our study demonstrates the differences between PPP and PV in both prevalence and clinical effect. Development of PPP occurred more often: in $0.66 \%$ of the patients with RA and in $1.73 \%$ of the patients with AS, compared to the general population, with a prevalence of $0.05 \%{ }^{6}$. In our study, only $0.99 \%$ of the patients with RA and $1.38 \%$ of the patients with AS developed biological-associated PV, while the prevalence in the general population is $2.0 \%$ and in $10.2 \%$ in the patients with $\mathrm{AS}^{7,8}$. However, the comparison between the prevalence found in our study and in the general population should be done with caution, because almost no well-defined population-based studies have been performed. Further, we confirm that PV and PPP are distinct from each other ${ }^{5}$. A remarkable finding is the significantly shorter time to onset for the PPP group compared to the PV group. Moreover, PPP and PV differ in the consequences for the treatment of RA

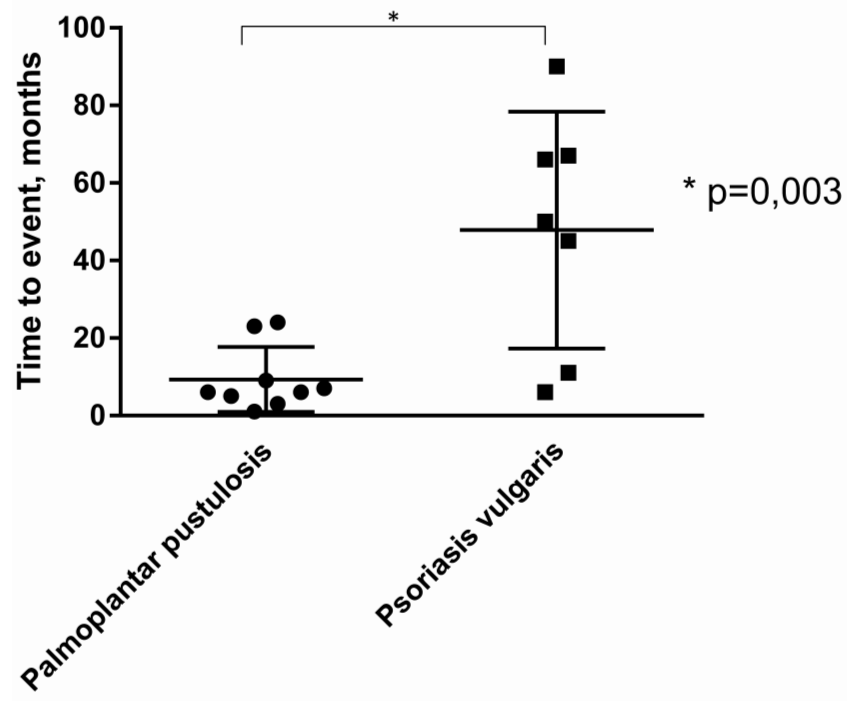

Figure 1. Differences in time to event in the development of palmoplantar pustulosis and psoriasis vulgaris.

and AS. Our data show that in most cases, discontinuation of their current biologic therapy is the only successful treatment of PPP. Because $80 \%$ of the patients developed an exacerbation while taking a second TNFi, they could be advised to switch to another mode of treatment. However, this should be studied in more detail.

The retrospective design of our study has some limitations. There could be an underestimation due to lack of registration in our database. However, all biologicals registered for the treatment of RA or AS were taken into account, when most studies focus only on TNFi. Further, this patient group is an excellent representation of the general RA and AS patient population, because of the large sample size of the Reade Rheumatology Registry, which reflects the standard clinical care. But most importantly, this is the first study in this field, to our knowledge, with a distinguished approach of patients who developed PPP or PV, and the differences found between PPP and PV emphasize the importance of studying both diseases differently to understand pathogenesis, optimal treatment, and the consequences for patients.

JILL RUWAARD, MD, Amsterdam Rheumatology and Immunology Center, location Reade; EVA R. VAN DER VLUGT, Amsterdam Rheumatology and Immunology Center, location Reade; GERTJAN WOLBINK, MD, PhD, Amsterdam Rheumatology and Immunology Center, location Reade, and the Department of Immunopathology, Sanquin Research and Landsteiner Laboratory Academic Medical Center, Amsterdam, the Netherlands. Dr. Wolbink received grant/research support from Pfizer. Address correspondence to Dr. J. Ruwaard, Amsterdam Rheumatology and Immunology Center, location Reade, Dr. Jan van Breemenstraat 2, $1056 \mathrm{AB}$ Amsterdam, the Netherlands.

E-mail: j.ruwaard@reade.nl

\section{REFERENCES}

1. Braun J, van den Berg R, Baraliakos X, Boehm H, Burgos-Vargas $\mathrm{R}$, Collantes-Estevez E, et al. 2010 update of the ASAS/EULAR recommendations for the management of ankylosing spondylitis. Ann Rheum Dis 2011;70:896-904.

2. Nam JL, Takase-Minegishi K, Ramiro S, Chatzidionysiou K, Smolen JS, van der Heijde D, et al. Efficacy of biological disease-modifying antirheumatic drugs: a systematic literature review informing the 2016 update of the EULAR recommendations for the management of rheumatoid arthritis. Ann Rheum Dis 2017;76:1113-36

3. Perez-Alvarez R, Perez-De-Lis M, Ramos-Casals M. 
Biologics-induced autoimmune diseases. Curr Opin Rheumatol 2013;25:56-64

4. Collamer AN, Battafarano DF. Psoriatic skin lesions induced by tumor necrosis factor antagonist therapy: clinical features and possible immunopathogenesis. Semin Arthritis Rheum 2010; 40:233-40.

5. de Waal AC, van de Kerkhof PC. Pustulosis palmoplantaris is a disease distinct from psoriasis. J Dermatolog Treat 2011;22:102-5.

6. Hellgren L, Mobacken H. Pustulosis palmaris et plantaris. Prevalence, clinical observations and prognosis. Acta Derm Venereol 1971;51:284-8.
7. Boehncke WH, Schon MP. Psoriasis. Lancet 2015;386:983-94.

8. de Winter JJ, van Mens LJ, van der Heijde D, Landewe R, Baeten DL. Prevalence of peripheral and extra-articular disease in ankylosing spondylitis versus non-radiographic axial spondyloarthritis: a meta-analysis. Arthritis Res Ther 2016;18:196.

First Release October 15 2018; J Rheumatol 2019;46:117-118; doi:10.3899/jrheum.180566 